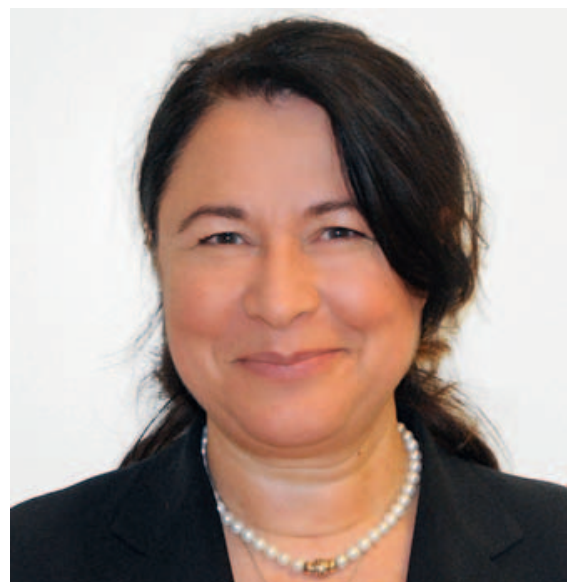

Die Klinik Arlesheim hat bei der ANQ-Qualitätsmessung sehr gut abgeschnitten. Was ist das für eine Messung?

Der Nationale Verein für Qualitätsentwicklung in Spitälern und Kliniken (ANQ) koordiniert und realisiert Qualitätsmessungen in der Akutsomatik, der Rehabilitation und der Psychiatrie. Die Resultate ermöglichen eine transparente und nationale Vergleichbarkeit. Aufgrund der Erkenntnisse aus den Messungen können Spitäler und Kliniken gezielt Massnahmen zur Verbesserung ihrer Qualität entwickeln. In den Jahren 2012 bis 2014 hat ANQ in den Schweizer Spitälern mit einem stationären psychiatrischen Angebot erstmalig schweizweit mit einheitlicher Vorgehensweise vergleichende Qualitätsdaten erhoben. Alle 64 Spitäler mit einer stationären Psychiatrie nahmen daran teil, auch unsere Klinik.

\section{Was genau zeigen die Anfang 2016 veröffentlichten ANQ-Ergebnisse?}

Es gibt einen Fragebogen von ANQ, der von den Patienten und den Ärzten

\title{
Anthroposophische Medizin bewährt sich auch in der Psychiatrie
}

Die Anfang 2016 veröffentlichten Qualitätsmessungen des Nationalen Vereins für Qualitätsentwicklung in Spitälern und Kliniken (ANQ) zeigen hervorragende Ergebnisse für die Anthroposophische Medizin, wie sie in der Klinik Arlesheim auch im Bereich Psychiatrie/Psychosomatik angewandt wird. Dr. med. Martina Haeck, Leiterin der Psychiatrie/Psychosomatik an der Klinik Arlesheim, berichtet im nachfolgenden Interview über ihren Ansatz in der Arbeit mit psychiatrischen Patientinnen und Patienten.

beim Eintritt in die Klinik und beim Austritt ausgefüllt wird. Der Vergleich der beiden Ergebnisse zeigt die Veränderung der Beschwerden (die Symptombelastung) zwischen Eintritt und Austritt. Da sowohl die Sicht der Patientinnen und Patienten als auch die der Behandelnden berücksichtigt wird, ergibt sich ein umfassendes Bild über die Behandlungsqualität. In der Auswertung von ANQ zeigt die Klinik Arlesheim hervorragende Ergebnisse, denen zufolge der Behandlungserfolg von den Patienten sogar noch höher beurteilt wird, als ihn die behandelnden Ärzte einschätzen. Bemerkenswert ist, dass die Verbesserung der Symptome mit dem Einsatz von weniger Psychopharmaka erreicht werden konnte - bei einem Kollektiv von bei Eintritt schwer kranken Patienten.

\section{Worauf führen Sie diese Ergebnisse zurück?}

Wir nehmen das als ein deutliches Zeichen, dass der ganzheitliche und integrative Ansatz der Anthroposophischen Medizin unseren Patientinnen und Patienten nützt. Ich bin überzeugt, dass unsere Medizin wirkt - das zeigt sich hier einmal mehr. Es spre- chen heute viele von Ganzheitlichkeit, auch von Individualität. Die Methodenvielfalt, die mir hier zur Verfügung steht, kommt den Patienten zugute und bereichert die individualisierte Medizin. Als ganz entscheidend sehe ich unseren Ansatz, wirklich individualisierte Medizin zu betreiben. Wir arbeiten mit dem Patienten zusammen, wir begleiten ihn entsprechend seinen Zielen. Das ist das Entscheidende: Es sind immer die Ziele des Patienten.

\section{Wie schaffen Sie das?}

Wir schauen, wie wir den Patienten am besten erreichen. Das können ganz unterschiedliche Kanäle sein. Der eine Patient ist mehr über Bilder zu erreichen, da ist dann auch eine eher bildhafte Sprache hilfreich. Den anderen müssen wir verstärkt über den Intellekt ansprechen.

In der Psychiatrie ist es ein weiter Weg, auf dem wir den Patienten begleiten. Vieles läuft über das Gespräch. Aber wir arbeiten auch mit dem Körper, mit den verschiedensten Sinnen, mit Bewegung und mit Rhythmus. Das sind wesentliche Bausteine unserer Therapie. 


\section{Können Sie das an einem Beispiel beschreiben?}

Hervorzuheben sind da insbesondere die Sinneswahrnehmungsübungen, man kann auch von Achtsamkeitsübungen sprechen. Von Achtsamkeit wird seit einigen Jahren vermehrt in der Medizin gesprochen - in der Klinik Arlesheim wird dies seit 1921 aktiv betrieben und weiterentwickelt. Ich möchte als Beispiel die Pflanzenbetrachtung nennen. Es reicht nicht zu sehen, dass Blumen auf dem Tisch stehen, sondern der Patient wird aufgefordert, die Blumen genau zu beschreiben: Was sind das für Blumen? Wie sehen sie aus? Wonach duften sie? Wie sind die Blätter strukturiert? Die Patientinnen und Patienten werden so über erlebte Sinneseindrücke und deren Beschreibung im Sinn von äusseren Wahrnehmungen darin unterstützt, sich selbst, also ihr Inneres besser wahrzunehmen. Das hilft ihnen auch, ihre Grenzen und Bedürfnisse $\mathrm{zu}$ sehen und zu berücksichtigen.

Jeder Morgen beginnt mit einer Morgenrunde. Die gesamte Patientengruppe geht zum Beispiel in den Park, um einen bestimmten Baum genauer zu betrachten oder um das Wetter zu beobachten. Es geht dabei immer um die Konzentration auf eine ganz bestimmte Aufgabe.

\section{Sie haben also auch Gruppen- angebote. Wie passt das zu der von Ihnen beschriebenen individuellen Medizin?}

Auch in der Gruppe kann das Individuelle seinen Platz haben. Ausserdem werden gerade auch durch die Interaktion in der Gruppe bestimmte Entwicklungen deutlich oder aber auch Punkte, an denen es weiterzuarbeiten gilt. Die Gruppentherapien erweisen sich als sehr hilfreich in der Ergänzung der individuellen Therapien, da die Patienten in geschütztem Rahmen voneinander profitieren können und auch für die Zeit nach dem Klinikaufenthalt üben.

Es ist noch gar nicht lange her, dass wir die Möglichkeit einer neuen Gruppenarbeit für die Psychoedukation geschaffen haben. Das ist eine Gruppentherapie für das stationäre und ambulante Setting nach einem stationären Aufenthalt, die sich sehr bewährt. In vierzehntägigem Abstand kommt die Gruppe zusammen und bespricht gemeinsam mit einer Ärztin/einem Arzt solche Themen wie Schlaf, Angst, Stressbewältigung und Selbstfürsorge.

\section{Sie erwähnten die \\ Methodenvielfalt - welche \\ Methoden stehen Ihnen zur Verfügung?}

Für jede Patientin, für jeden Patienten stellen wir ein individuelles Therapieprogramm zusammen. Unsere Physiotherapie konzentriert sich auf die spezifisch anthroposophischen Therapien wie die rhythmische Massage und verschiedene rhythmische Bäder. Auch in diesen Therapien geht es um den Rhythmus - ein ganz zentraler Ansatz, denn «Rhythmus trägt Leben». Man kann den Rhythmus als Heilmittel bezeichnen - in den Therapien, in den rhythmischen Prozessen der Heilmittelherstellung, aber auch in der Gestaltung des Tages- und Wochenablaufs, wodurch die Tage strukturiert sind und dadurch einen gewissen Halt bieten.

Die künstlerischen Therapien - die Musiktherapie, die therapeutische Sprachgestaltung und das therapeutische Malen und Plastizieren - sowie die Heileurythmie beinhalten ebenfalls viel Rhythmisches. Sie bieten den Patienten mit den Übungen ein Instrumentarium an die Hand, sodass sie selbst und aktiv in den Gesundungsprozess eingreifen können. Das halte ich für sehr wesentlich - dass der Patient merkt, das Steuer wieder selbst in die Hand nehmen zu können.

\section{Sie setzen weniger Psychopharmaka ein, aber Sie nutzen auch diese Mittel?}

Ja, ganz klar. Es gibt Krankheitsbilder und individuelle Situationen, da geht es gar nicht ohne. Wir sind froh, zur Verfügung zu haben, was durch die medizinische Forschung erreicht wurde und dass wir auf verschiedenste pharmakologische Möglichkeiten zurückgreifen können. Die Anthroposophische Medizin setzt auch unmittelbar auf der Schulmedizin auf. Das ist ja gerade das Wunderbare: Wir haben alle Möglichkeiten der modernen Medizin, erweitern diese aber durch den ganzheitlichen Ansatz der anthroposophischen Menschenkunde und können so eine erweiterte Schulmedizin anbieten, die im echten Sinn integrativ ist.

\section{Wie wird sich Ihre Psychiatrie weiterentwickeln?}

Die oben angesprochenen Ergebnisse sind die der ANQ-Qualitätsmessung. Da geht es um die Verbesserung der Symptome, man kann klar sagen, es ist ein Wirksamkeitsnachweis eines Gesamtkonzepts. Unabhängig davon holen wir selbstverständlich immer wieder Feedback von unseren Patientinnen und Patienten ein. Durch die Zufriedenheitsmessungen erfahren wir, was sie von uns brauchen. Das setzen wir entsprechend unserer Möglichkeiten um.

Und wir beziehen natürlich auch die Entwicklung aus Medizin und Psychiatrie ein. Die Ergebnisse der heutigen Forschung werden von uns berücksichtigt.

\section{Wie sieht es mit Ihrer eigenen Forschung aus?}

Die Klinik Arlesheim hat seit einigen Jahren eine eigene Forschungsabteilung, die sich insbesondere auf sol- 
che Themen wie Rhythmusforschung konzentriert, z.B. auf Arbeiten mit der Methodik der Herzfrequenz-Variabilitätsforschung. Es geht ja darum, dass wir für die Komplementärmedizin, insbesondere auch die Anthroposophische Medizin, Wirksamkeitsnachweise benötigen. Dafür brauchen wir Forschungsergebnisse. Da gibt es bei uns eine ganze Reihe spannender Themen und Fragen, die erforscht werden wollen. Notwendig ist hier die Beantragung von Fördergeldern, also sogenannten Drittmitteln.
Welche Schwerpunkte sehen Sie noch für die Zukunft?

Ein Schwerpunkt ist ganz sicher die Ausbildung. Der Fachkräftemangel macht auch vor unserer Klinik nicht halt. Und wir benötigen für unsere Arbeit speziell ausgebildete Fachkräfte. Also müssen wir uns auch für unseren Nachwuchs engagieren und Ausbildungsmöglichkeiten schaffen.

Aktuell haben wir einen grossen Meilenstein erreicht, indem wir die Weiterbildungsermächtigung im Fach- bereich Psychiatrie erhalten haben. Neu können unsere Assistenzärzte zwei Jahre auf unserer Psychiatriestation für ihre Facharztausbildung anerkennen lassen. Das ist auch für uns ein echter Gewinn, da die Begleitung unserer Patientinnen und Patienten deutlich mehr Kontinuität erhält.

Liebe Frau Dr. Haeck, haben Sie herzlichen Dank für das Gespräch und viel Erfolg für Ihre weitere Arbeit! 